\title{
Radiation Hydrodynamical Models for Type I Superluminous Supernovae: Constraints on Progenitors and Explosion Mechanisms
}

\author{
Ken'ichi Nomoto ${ }^{1,6}$, Alexey Tolstov ${ }^{1}$, Elena Sorokina ${ }^{2}$, \\ Sergei Blinnikov ${ }^{3,1}$, Melina Bersten ${ }^{4,1}$ and Tomoharu Suzuki ${ }^{5}$ \\ ${ }^{1}$ Kavli Institute for the Physics and Mathematics of the Universe (WPI), \\ The University of Tokyo, Kashiwa, Chiba 277-8583, Japan \\ ${ }^{2}$ Sternberg Astronomical Institute, M.V.Lomonosov Moscow State U., 119991 Moscow, Russia \\ ${ }^{3}$ Institute for Theoretical and Experimental Physics, 117218 Moscow, Russia \\ ${ }^{4}$ Instituto de Astrofisica La Plata, 1900 La Plata, Argentina \\ ${ }^{5}$ College of Engineering, Chubu University, Kasugai, Aichi 487-8501, Japan \\ ${ }^{6}$ Hamamatsu Professor \\ email: nomoto@astron.s.u-tokyo.ac.jp
}

\begin{abstract}
The physical origin of Type-I (hydrogen-less) superluminous supernovae (SLSNe-I), whose luminosities are 10 to 500 times higher than normal core-collapse supernovae, remains still unknown. Thanks to their brightness, SLSNe-I would be useful probes of distant Universe. For the power source of the light curves of SLSNe-I, radioactive-decays, magnetars, and circumstellar interactions have been proposed, although no definitive conclusions have been reached yet. Since most of light curve studies have been based on simplified semi-analytic models, we have constructed multi-color light curve models by means of detailed radiation hydrodynamical calculations for various mass of stars including very massive ones and large amount of mass loss. We compare the rising time, peak luminosity, width, and decline rate of the model light curves with observations of SLSNe-I and obtain constraints on their progenitors and explosion mechanisms. We particularly pay attention to the recently reported double peaks of the light curves. We discuss how to discriminate three models, relevant models parameters, their evolutionary origins, and implications for the early evolution of the Universe.
\end{abstract}

Keywords. magnetar, stellar mass loss, supernovae, superluminous supernovae

\section{Superluminous Supernovae}

Superluminous supernovae (SLSNe) are brighter than -21 magnitude in any optical band at the maximum brightness, which is $\approx 30$ times brighter than the average of normal supernovae (Quimby et al. 2012, Gal-Yam et al. 2012). They are divided into Type I (SLSN-I) and Type II (SLSN-II) according to the absence and presence of hydrogen feature in the spectra. It is evident that there is significant dispersion in both rise and decay time scales. These differences could indicate some diversity in the progenitors of SLSNe.

SLSNe-II, like SN 2006gy, may be powered by interactions of the SN ejecta with CSM in the form of steady wind or a shell (Moriya et al. 2013). The origin of SLSNe-I is still a matter of debate (Gal-Yam et al. 2012). Currently three possibilities are under consideration (a) pair instability supernovae, (b) supernovae powered by ejecta/wind interaction, and (c) supernovae powered by a spin down of a rapidly rotating young magnetar. In the present paper, we focus on the magnetar and circumstellar interaction models for SLSNe-I and discuss possible engines powering the observed super-luminosities. 


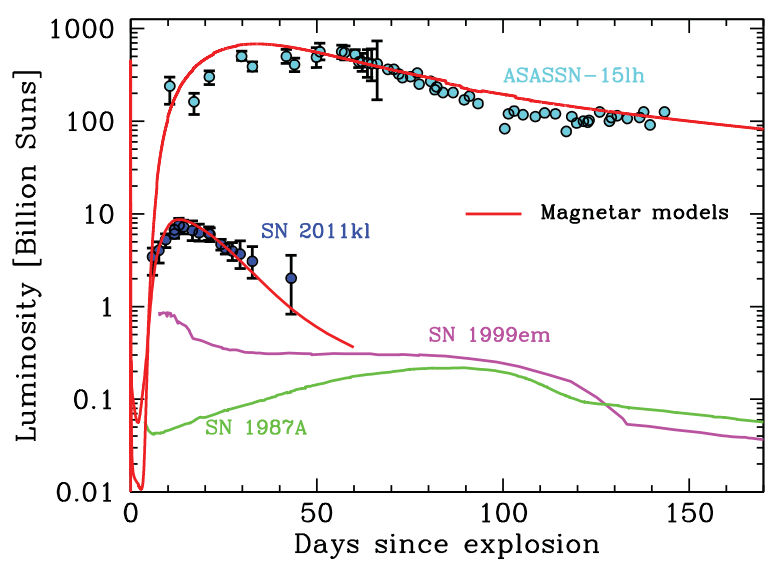

Figure 1. The light curves of ASASSN-15lh and SN 2011kl compared with SN 1999em and SN 1987A, and with the magnetar models (Bersten et al. 2016).

The existence of SLSNe opens the possibility of studying extremely luminous supernovae in the very early Universe. Understanding their nature will open new paths to better comprehend the origin and evolution of stars in the Universe.

\section{Magnetar Driven Supernovae}

A rapidly rotating neutron stars with large magnetic fields, $\approx 10^{14}-10^{15}$ Gauss, or as usually called "magnetar" could be an extra source to power the light curve of SNe (see, e.g., Bersten et al. 2016 for references). Important question is if the rotation energy of the magnetar is efficiently deposited into the supernova ejecta.

SN 2011kl represents the first SLSN associated with an ultra long GRB (Greiner et al. 2015). This gamma-ray burst lasted several hours whereas the typical long-duration GRBs (LGRBs) fade in a matter of minutes. A handful of SNe have been observed coincident with LGRB but SN 2011kl was more than three times more luminous than normal supernovae. The peculiar properties of both the LGRB and the supernova suggest a common extreme nature.

ASASSN-15lh is possibly the most luminous and powerful explosion ever seen (Dong et al. 2016). It was 200 times more powerful than a typical supernova. And it outshone all of the other SLSNe by at least a factor of two. During over one month its luminosity was twenty times larger than that of the whole Milky Way galaxy with its billions of stars. Such an enormous release of energy has challenged the current models that attempt to explain the SLSN phenomenon.

The spectra of both SN 2011kl and ASASSN-15lh showed no evidence of hydrogen, i.e., these are SLSNe-I. Bersten et al. (2016) have analyzed two mysterious cases among SLSNe. Despite the differences between them, both events could be explained as explosions boosted by a highly magnetized, rapidly spinning neutron star (magnetar).

Bersten et al. (2016) have performed numerical hydrodynamical calculations to explore the magnetar hypothesis for these two intriguing objects. They found that both SLSNe could be understood in the framework of magnetar-powered supernovae (Fig. 1). In particular for the extreme ASASSN-15lh, they were able to find a magnetar source with physically allowed properties of magnetic field strength and rotation period. The solution avoided the prohibited realm of neutron-star spins that would cause the object to 
breakup due to centrifugal forces. To confirm the proposed model, it is critical to obtain further observations when the material ejected by the supernova is expected to become thin.

The magnetar models above were performed to reproduce observations of ASASSN15 lh until $\sim 150$ days. However, recent publications shows that ASASSN-15lh has undergone an UV re-brightening (Brown, et al. 2016, Godoy-Rivera et al. 2016). The long and bright UV plateau of 120 days duration (Godoy-Rivera et al. 2016) could be in contradiction with a purely magnetar source. An interesting possibility could be to invoke a combination of multiple power sources (including circumstellar interaction) (Chatzopoulos et al. 2016, Tolstov et al. 2017).

\section{Circumstellar Interaction Models}

SN light curves for circumstellar interacting models have been constructed analytically for both optically thin and optically thick cases in a number of papers (Chevalier et al. 2011, Moriya et al. 2013, Chatzopoulos et al. 2013). However, radiation hydrodynamical models are noticeably different from the analytic models (Sorokina et al. 2016).

Sorokina et al. (2016) calculated radiation-hydrodynamical models of circumstellar interaction to reproduce LCs of both SN 2010gx and PTF09cnd for optically thick CSM. PTF09cnd and SN 2010gx reach peak luminosities too high to be explained by the ${ }^{56} \mathrm{Ni}$ production allowed by their late time photometric limits (Pastorello et al. 2010). For the LC shape, SN 2010gx has one of the narrowest LC while PTF09cnd has one of the broadest LC among SLSNe-I.

In the models by Sorokina et al. (2016), when the shock wave structure starts to form due to collision between the ejecta and the CSM, the envelope is cool and transparent. Then the emission from the shock front heats the gas in the envelope, thus making it opaque, and the photosphere moves to the outermost layers rather quickly. When the photospheric radius reaches its maximum, one can observe maximal emission from the supernova.

The speed of the growth of the photospheric radius depends on the mass of the envelope, since more photons must be emitted from the shock to heat larger mass envelopes. This is why the photosphere moves to the outermost layers in model N0 faster than in model B0. At the beginning of this post-maximum stage all gas in the envelope is already heated by the photons which came from the shock region and diffused through the envelope to the outer edge, and the whole system (ejecta and envelope) becomes almost isothermal. Model N0 seems to provide the best match to SN 2010gx. The maximmu emission and the slopes of the light curves after the maximum are in excellent agreement.

Model B0 is the best for PTF09cnd. For the models with a massive envelope the rising time is determined by diffusion time of the photons from the shock wave and by the movement of the photosphere to the outermost edge of the envelope. The rising time which more or less fits the observations was obtained for the model B0 with $49 \mathrm{M}_{\odot}$ in the envelope and $5 \mathrm{M}_{\odot}$ in the ejecta of the explosion with the energy of $4 \cdot 10^{51}$ ergs.

For these SNe, moderate explosion energy $E_{51} \sim 2-4$ is enough to explain both LCs. Main difference between the two SLSNe are the masses of CSM. Only $\sim 5 M_{\odot}$ of non-H (He, C, O) is needed to reproduce SN 2010gx light curve (Fig. 2), while almost $55 M_{\odot}$ CSM to reproduce the broad LC of PTF09cnd (Fig. 3). 


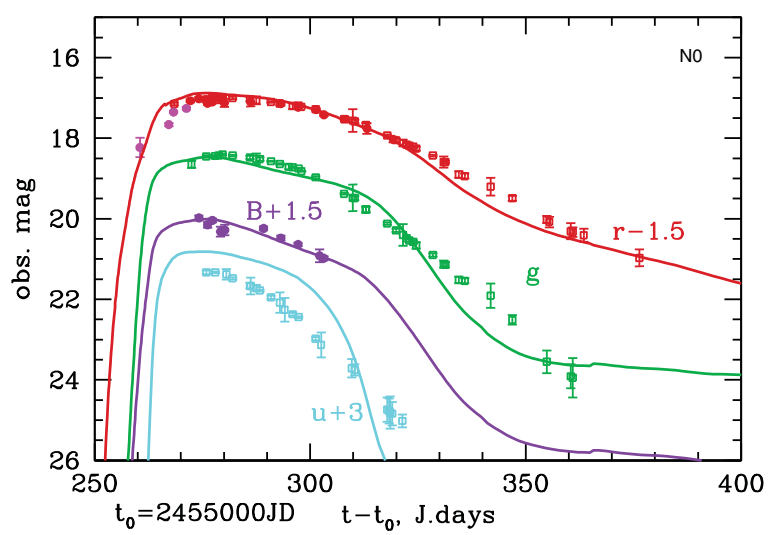

Figure 2. Synthetic light curves for SN 2010gx, in $r, g, B$, and $u$ filters compared with Pan-STARRS and PTF observations (Sorokina et al. 2016). Pan-STARRS points are designated with open squares ( $u, g$, and $R$ bands), PTF points, with filled circles ( $B$ and $r$ bands). Four pink points in the beginning of the $r$ band shows PTF observations in the Mould $R$-band which is similar to the SDSS $r$ band.

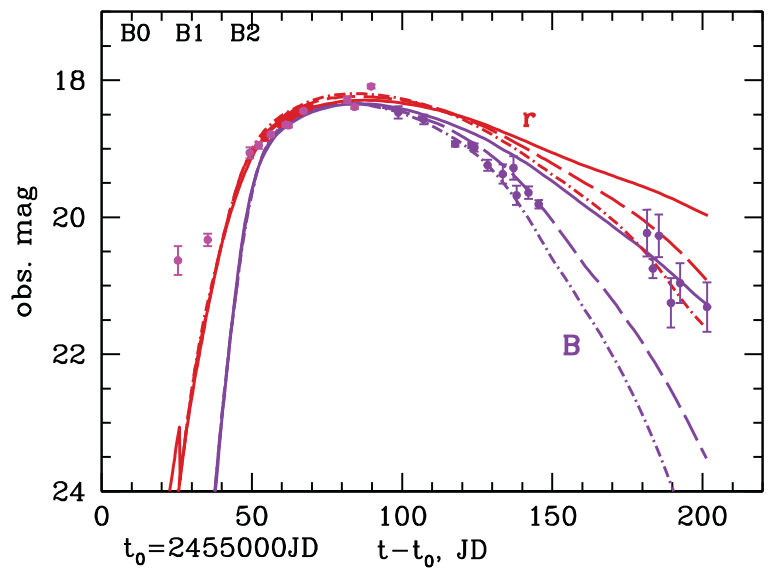

Figure 3. Observed (dots) and synthetic (lines) light curves for PTF09cnd in $r$ and $B$ filters . The solid, dashed, and dash-dotted lines correspond to the models with the static outer envelope, expanding envelope, and faster expanding envelope, respectively (Sorokina et al. 2016).

\section{Origin of Circumstellar Matter}

SN ejecta-CSM interaction is a possible energy source for SLSNe-I, if the CSM is depleted of its hydrogen. Evolutionary calculations of very massive stars $\left(\gtrsim 100 M_{\odot}\right)$ of different metallicities show that most of $\mathrm{H}$ burns into He during main sequence before the intensive mass loss (Yusof et al. 2013). Such H-burning occurs in a very extended convective core that reaches outermost layers of the star. In addition, the rotation of the star forms a meridional circulation which also helps $\mathrm{H}$ (and, possibly, some part of $\mathrm{He}$ ) to be burned even near the surface. In later phase, the star undergoes a loss of a large amount of envelope which is no longer $\mathrm{H}$-rich, but $\mathrm{He}$ with enhanced $\mathrm{C}+\mathrm{O}$. This could be a plausible way to produce $\mathrm{CSM}$ of $\sim 50 M_{\odot}$ He with enhanced $\mathrm{C}+\mathrm{O}$.

Materials cast off by instabilities in the cores of very massive stars in their final years, e.g., pulsational pair-instability (Woosley et al. 2007, Ohkubo et al. 2009), are possible sources of $\mathrm{C}+\mathrm{O}$ CSM. 
In close binary systems, a merger (or even multiple mergers) of the $\mathrm{C}+\mathrm{O}$ core from an evolved WR star with another hydrogen-deficient star, like a neutron star, a white dwarf, or WR star could occur (Taam et al. 2000, Glebbeek et al. 2009, Chevalier et al. 2012). Such processes could form a dense C+O CSM or common envelope. In a dense stellar cluster, the (multiple) collision of massive stars (in particular, Wolf-Rayet stars) can make a massive $\mathrm{C}+\mathrm{O}$ star surrounded by a massive non-Hydrogen CSM (Portegies Zwart et al. 2007, Suzuki et al. 2007).

The result of those events could be an envelope or an expanding wind with the size large enough to provide the long living shock wave. It may break out through the envelope in a few months. The explosion within such an envelope could give rise to an SLSN-I.

The work has been supported by the World Premier International Research Center Initiative (WPI), MEXT, Japan, and by the Grants-in-Aid for Scientific Research of the JSPS (JP26400222, JP16H02168, JP17K05382).

\section{References}

Bersten, M., Benvenuto, O., Orellana, M., \& Nomoto, K. 2016, ApJ 817, L8

Brown, P. J., Yang, Y., Cooke, J., et al. 2016, ApJ 828, 3

Chatzopoulos, E., et al. 2013, ApJ 773, 76

Chatzopoulos, E., Wheeler, J. C., Vinko, J., et al. 2016, ApJ 828, 94

Chevalier, R. A. \& Irwin, C. M. 2011, ApJ (Letters) 729, L6

Chevalier, R. A. 2012, ApJ (Letters) 752, L2

Dong, S., Shappee, B. J., Prieto, J. L., et al. 2016, Science 351, 257

Gal-Yam, A. 2012, Science 337, 927

Godoy-Rivera, D., Stanek, K. Z., Kochanek, C. S., et al. 2016, MNRAS 466, 1428

Glebbeek, E., Gaburov, E., de Mink, S. E., et al. 2009, A\& A 497, 255

Greiner, J., Mazzali, P. A., Kann, D. A., et al. 2015, Nature 523, 189

Moriya, T., et al. 2013, MNRAS 428, 1020

Ohkubo, T., Nomoto, K., Umeda, H., Yoshida, N., \& Tsuruta, S. 2009, ApJ 706, 1184

Pastorello, A., Smartt, S., Botticella, M., et al. 2010, ApJ (Letters) 724, L16

Portegies Zwart S. F., van den Heuvel E. P. J. 2007, Nature 450, 388

Quimby, R. 2012, IAU Symp. 279, Death of Massive Stars: Supernovae and Gamma-Ray Bursts, ed. P. Roming, et al., 22

Sorokina, E., Blinnikov, S., Nomoto, K., Quimby, R., \& Tolstov, A. 2016, ApJ 829, 17

Suzuki, T. K., Nakasato, N., Baumgardt, H., et al. 2007, ApJ 668, L19

Taam, R. E. \& Sandquist, E. L. 2000, ARAA 38, 113

Tolstov, A., Nomoto, K., Blinnikov, S., Sorokina, E., Quimby, R., \& Baklanov, P. 2017, ApJ 835, 266

Woosley, S. E., Blinnikov. S. \& Heger, A. 2007, Nature 450, 390

Yusof, N., Hirschi, R., Meynet, G., et al. 2013, MNRAS 433, 1114 